\title{
Using Transthoracic Echocardiography to Predict Fluid Responsiveness after Passive Leg Raising Test: Caution Needed
}

\author{
Natesh Prabu $\mathrm{R}^{1}$, Amol T Kothekar ${ }^{2}$ \\ Keywords: Passive leg raising, Transthoracic echocardiography, Velocity-time integral. \\ Indian Journal of Critical Care Medicine (2020): 10.5005/jp-journals-10071-23646
}

We read with interest the article by El-Nawawy et al., ${ }^{1}$ on the accuracy of passive leg raising test (PLR) in the prediction of fluid responsiveness (FR) in children. The authors have demonstrated the prediction of FR using PLR with reasonable accuracy. The study is of interest since there is a paucity of information on the utility of PLR in children.

We would like to raise a couple of concerns. First, performing PLR in infants and small children may be challenging. Unlike adults, these groups of patients may not cooperate for the procedure. The authors have not mentioned if they had to exclude any patient due to non-cooperation or other reasons. In a recent study looking at PLR in infants and children (mean age was $85.8 \pm 69.5$ months), $<40 \%$ of patients could complete the PLR protocol. ${ }^{2}$ Second, we have concerns regarding the use of transthoracic echocardiography (TTE) to track the changes in velocity-time integral (VTI)/stroke volume (SV). The authors have used the left ventricular outflow tract velocity-time integral (LVOT-VTI) obtained by TTE for measurement of SV/cardiac output (CO) in response to PLR. Based on the results of the study, authors suggest the use of change in stroke volume $(\Delta \mathrm{SV}) \geq 10 \%$ or change in cardiac index $(\Delta \mathrm{Cl})>8.7 \%$ after PLR as a cut-off for identifying fluid responder from non-responders.

Cardiac output and SV measurements using VTI obtained by TTE have its limitations apart from being non-continuous. ${ }^{3}$ The VTI measurements are subject to individual variation due to various reasons. First, it requires skill to obtain an optimal image in which the ultrasound beam is aligned with cardiac structures especially obtaining the alignment of the Doppler angle parallel to blood flow. Second, it is challenging to maintain the probe in the same position while repeating the measurements during PLR. Any change in hand position during PLR can cause an error that will affect the measurements. Moreover, there can be a change in VTI due to arrhythmias or changes in rate and depth of ventilation and the readings should be measured at the same period of respiration (end-expiration) especially while comparing the results before and after an intervention. The authors have not mentioned about the hand position, period of respiration when the measurement was taken. All these factors have the potential to affect the measurements during PLR confounding its results.

The authors mention good intraobserver reliability in three baseline measurements of SV with an average intraclass correlation of 0.93 [95\% confidence interval $(\mathrm{Cl})=0.91$ to 0.95 ], $p<0.001$ in a pilot study. However, when we are looking at serial measurements, knowing the precision and the least significant change (LSC) of VTI between examinations is probably more important. In a study looking specifically at LSC and precision of repeated values by same
${ }^{1}$ Department of Critical Care Medicine, St John's Medical College Hospital, Bengaluru, Karnataka, India

${ }^{2}$ Department of Anesthesia, Critical Care and Pain, Tata Memorial Center, Homi Bhabha National Institute, Mumbai, Maharashtra, India

Corresponding Author: Amol T Kothekar, Department of Anesthesia, Critical Care and Pain, Tata Memorial Center, Homi Bhabha National Institute, Mumbai, Maharashtra, India, e-mail: amolkothekar@yahoo. com

How to cite this article: Prabu RN, Kothekar AT. Using Transthoracic Echocardiography to Predict Fluid Responsiveness after Passive Leg Raising Test: Caution Needed. Indian J Crit Care Med 2020;24(11): 1147-1148.

Source of support: Nil

Conflict of interest: None

and different operators, Jozwiak et al. ${ }^{4}$ have demonstrated a lack of precision of VTI for assessing the effects of tests predicting preload responsiveness. Jozwiak et al. showed that the LSC was $11 \%$ for VTI between two successive examinations performed by the same operator and $14 \%$ if two operators performed the examinations. This means that it is difficult for a single operator to identify with precision a change in $\mathrm{VTI} \leq 10 \%$ questioning the use of VTI obtained by TTE for the identification of small changes in $\mathrm{CO}$ required in PLR. The cut-off values quoted in the study by El-Nawawy et al. (10 and $8.7 \%$ for SV change and cardiac index change) are lower than the LSC of VTI limiting its applications. This is a real concern while using VTI-TTE.

In conclusion, the lack of precision of VTI values $<11 \%$ has been demonstrated in adults. In children $<5$ years where PLR is yet to be established, studies using VTI for PLR should be interpreted with caution due to the limitation of the absence of precision studies of VTI in this age group.

\section{References}

1. El-Nawawy AA, Farghaly PM, Hassouna HM. Accuracy of passive leg raising test in prediction of fluid responsiveness in children. Indian J Crit Care Med 2020;24(5):344-349. DOI: 10.5005/jp-journals-1007123432.

2. Chaiyaphruk M, Boonjindasarp W, Sritippayawan S, Deerojanawong J, Samransamruajkit R. Abstract P-033: accuracy of passive leg raising test in predicting of fluid responsiveness in children on ultrasonic cardiac output monitoring (USCOM ${ }^{\circledR}$ ). Pediatr Crit Care Med 2018;19:58. DOI: 10.1097/01.pcc.0000537490.01142.20. 
3. Blanco P. Rationale for using the velocity-time integral and the minute distance for assessing the stroke volume and cardiac output in point-of-care settings. Ultrasound J 2020. 12. DOI: 10.1186/s13089020-00170-x.
4. Jozwiak M, Mercado P, Teboul JL, Benmalek A, Gimenez J, Dépret F, et al. What is the lowest change in cardiac output that transthoracic echocardiography can detect? Crit Care 2019;23(1):1-10. DOI: 10.1186/ s13054-019-2413-x. 